Supporting Information for

\title{
Interfacial Strain Engineering in Wide-Bandgap GeS Thin Films for Photovoltaics
}

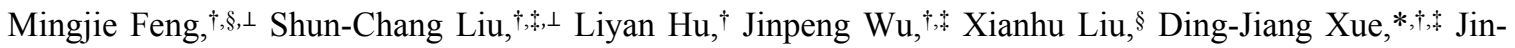
Song $\mathrm{Hu}^{\dagger, *}$, and Li-Jun Wan ${ }^{\dagger, \$}$

†Beijing National Laboratory for Molecular Sciences (BNLMS), CAS Key Laboratory of Molecular Nanostructure and Nanotechnology, Institute of Chemistry, Chinese Academy of Sciences, Beijing 100190, China

$\S$ National Engineering Research Center for Advanced Polymer Processing Technology, Zhengzhou University, Zhengzhou 450002, China

¥University of Chinese Academy of Sciences, Beijing 100049, China

${ }^{\perp}$ These authors contributed equally.

* To whom correspondence should be addressed. E-mail: djxue@iccas.ac.cn

This file includes Experiment Section, Figures S1-S13, and Tables S1-S2. 


\section{Experimental Section}

GeS Film Deposition. The soda lime glass (SLG) substrates were ultrasonically cleaned with deionized water, acetone, and isopropanol for about 40 minutes followed by drying with $\mathrm{N}_{2}$ gas. Then, the bilayer Mo back contacts with a total thickness about $400 \mathrm{~nm}$ was made on SLG substrates by a two-step magnetron sputtering process. The first layer was deposited by magnetron sputtering under a high working pressure (7 mTorr) at $2 \mathrm{~W} \mathrm{~cm}^{-2}$ power density for $20 \mathrm{~min}$; the second layer was deposited by magnetron sputtering under 3 mTorr pressure at $5 \mathrm{~W} \mathrm{~cm}^{-2}$ power density for $30 \mathrm{~min}$. Subsequently, prior to the deposition of $\mathrm{GeS}$, the surface of Mo was selenized at $600^{\circ} \mathrm{C}$ to form $\mathrm{MoSe}_{2}$ layer. Through a previously reported close-space sublimation (CSS) method, ${ }^{[1]} \mathrm{GeS}$ absorber layers were deposited on the selenized Mo-coated glass. Specifically, GeS crystalline powder ( $0.35 \mathrm{~g}$, 99.999\%, Jiangxi Ketai Advanced Materials Co. Ltd.) was put onto the cleaned SLG, which was loaded on top of the AlN plate and had a distance of $0.8 \mathrm{~cm}$ with substrate. When the pressure was below 10 mTorr, the CSS program would start to run. First, the source and substrate were preheated at $390{ }^{\circ} \mathrm{C}$ for $10 \mathrm{~min}$, then rapidly heated to $480{ }^{\circ} \mathrm{C}$ within $15 \mathrm{~s}$, and maintained at this temperature for $10 \mathrm{~s}$. Finally, nitrogen was injected into the deposition chamber, and the film was annealed at the same temperature $\left(480{ }^{\circ} \mathrm{C}\right)$ for 5 min. During the whole deposition process, graphite blocks with high heat capacity were covered on the substrate, which was adopted to stabilize the substrate temperature. Amorphous GeS films were deposited by a modified close-space sublimation method. There was no preheating process for the substrate, thereby leading to the deposition of amorphous GeS arising from the high crystallization temperature of GeS. The source was rapidly heated to $350{ }^{\circ} \mathrm{C}$ within $15 \mathrm{~s}$, maintained this temperature for $30 \mathrm{~s}$, and finally turned off the heating. The amorphous GeS powder was then obtained by scraping from the as-prepared amorphous GeS films.

Solar Cell Fabrication. The full GeS thin-film solar cell had a structure of glass/Mo/MoSe $2 / \mathrm{GeS} / \mathrm{CdS} / \mathrm{i}-\mathrm{ZnO} / \mathrm{ITO} / \mathrm{Ag}$. The GeS layer was deposited by CSS method described above. CdS buffer layer was deposited on the GeS layer by magnetron sputtering according to previous reports. ${ }^{[2]}$ Window layers of $\mathrm{i}-\mathrm{ZnO}$ and ITO were successively deposited by magnetron sputtering. The top Ag grid electrodes were finally deposited by 
thermal evaporation. The active area of the devices was $0.23 \mathrm{~cm}^{2}$ defined by mechanical scribing.

Materials and Device Characterization. The crystal structure of GeS films was determined by powder X-ray diffraction (Regaku, D/Max-2500 diffractometer equipped with a $\mathrm{Cu} \mathrm{K} \alpha_{1}$ radiation, $\lambda=1.54056 \AA$ ). Differential thermal analysis (DTA) was characterized by PerkinElmer Instruments, Diamond TG/DTA6300. The surface morphology was obtained using a Hitachi S-4800 scanning electron microscopy (SEM). The optical transmittance of GeS film was recorded via UV-vis-near IR spectrophotometer (UH4150, HITACHI). Ultraviolet photoemission spectroscopy (UPS, Specs UVLS, He I excitation, $21.21 \mathrm{eV}$, referenced to the Femi edge of argon etched gold) was employed to detect the Fermi level and valence band of GeS film. Raman spectrum (Horiba JobinYvon, LabRAM HR800) was measured under the excitation line of $532 \mathrm{~nm}$. The current densityvoltage (J-V) curves of photovoltaic devices were measured by a solar simulator (Newport, USA) equipped with $450 \mathrm{~W}$ xenon lamp (OSRAM) and a Keithley-2420 source meter. Light intensity was adjusted using a NREL certified Si solar cell with a $\mathrm{KG}^{-2}$ filter for approximating AM 1.5G light $\left(100 \mathrm{~mW} \mathrm{~cm} \mathrm{~cm}^{-2}\right)$. Two indoor light sources were a fluorescent tube (Philips T5 $6500 \mathrm{~K}$ ) and a white LED (PAK $4101924000 \mathrm{~K}$ ). The lamps were adjusted to an appropriate height to achieve an illuminance of 1000 lux on the solar cell surface. The illuminance and corresponding irr adiance were measured using an illuminance meter (TES-1334A) and an optical power meter (Thorlabs PM400). The external quantum efficiency (EQE) was measured with a lock-in amplifier. Light source was generated by a $300 \mathrm{~W}$ xenon lamp of Newport (Oriel, 69911, Newport Corporation, Irvine, CA, USA) and then was split into specific wavelength using Newport Oriel cornerstone 130 1/8 monochromator (Oriel, model 74004, Newport Corporation, Irvine, CA, USA).

Calculation methods. The density of states (DOS) of the nine materials were calculated based on the general gradient approximation (GGA) ${ }^{[3]}$ combined with the Perdew-BurkeErnzerhofer (PBE) formula by using the Vienna ab initio simulation package (VASP). ${ }^{[4,5]}$ The ultrasoft pseudopotential was used to describe the exchange-correlation effects and electron-ion interactions. An energy cutoff of $600 \mathrm{eV}$ was set for the plane-wave basis, and the Brillouin-Zone (BZ) integrals ${ }^{[6]}$ was sampled by a $6 \times 6 \times 1$ Monkhorst-Pack k-points set. 
For the lattice relaxations, the maximum force criterion and perpendicular force were all set as $0.01 \mathrm{eV} / \AA \AA$.
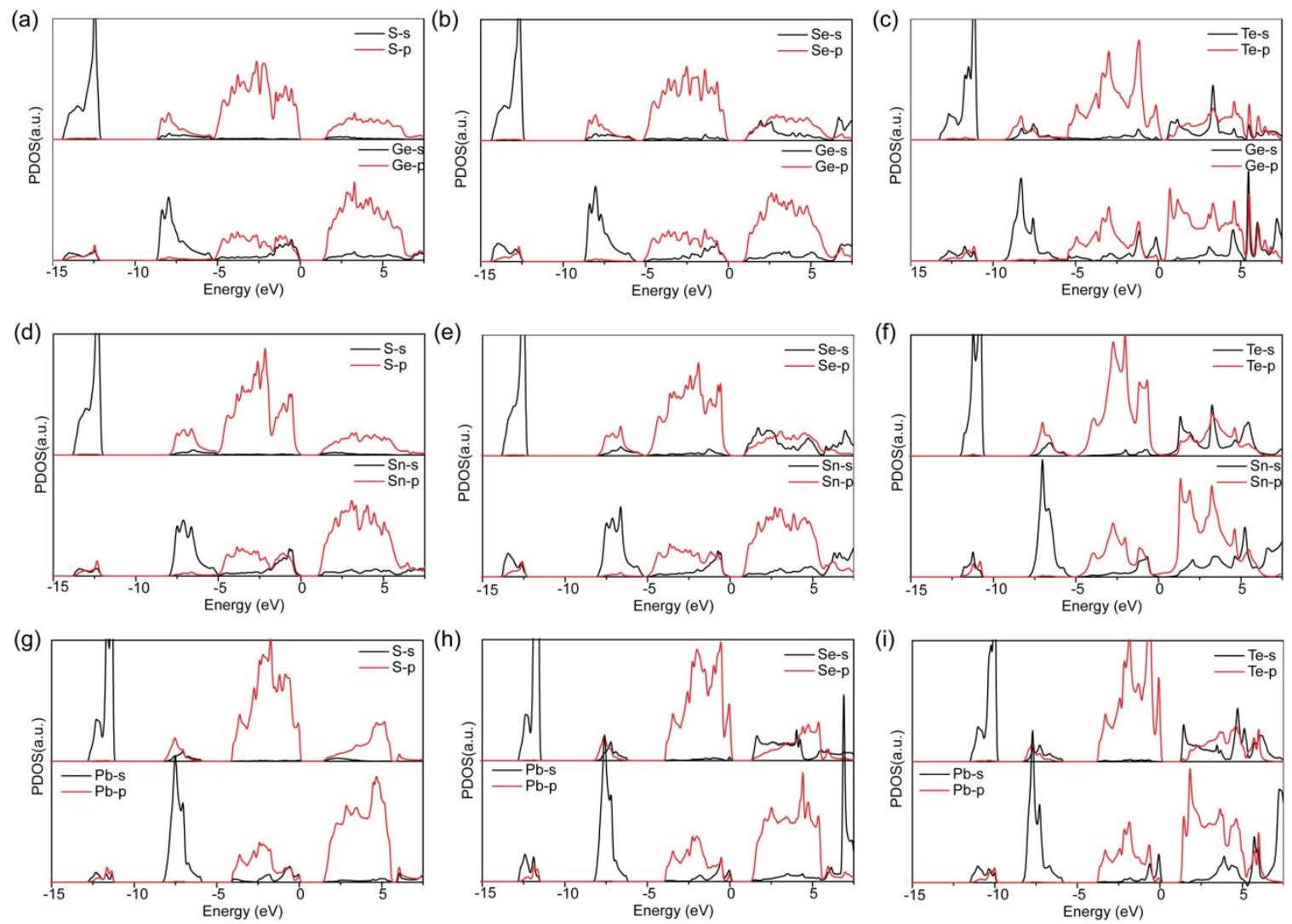

Figure S1. Calculated partial density of states (PDOS) of (a) GeS, (b) GeS, (d) SnS and (e) SnSe with Pnma phase, and (c) GeTe, (f) SnTe, (g) PbS, (h) PbSe and (i) PbTe with $\mathrm{NaCl}$ phase. 


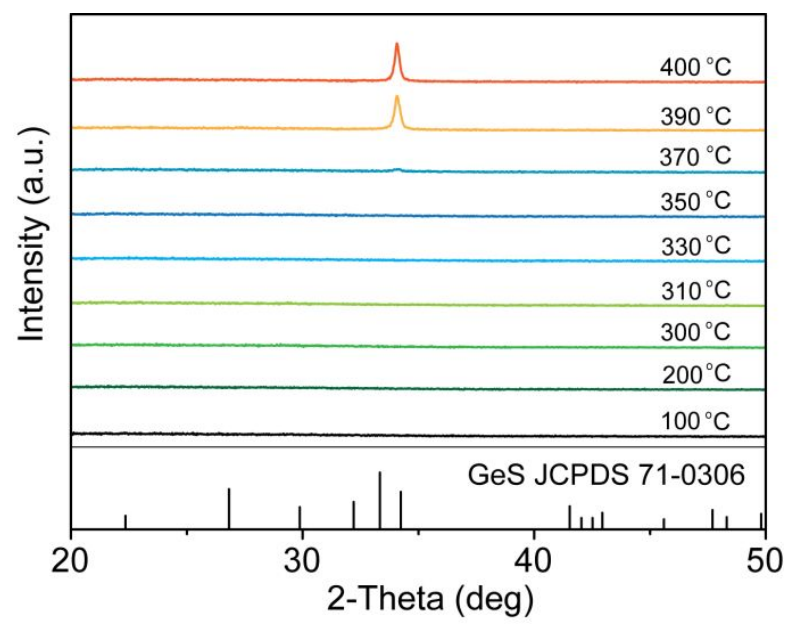

Figure S2. Temperature-dependent XRD patterns of a-GeS powder from 100 to $400{ }^{\circ} \mathrm{C}$. 

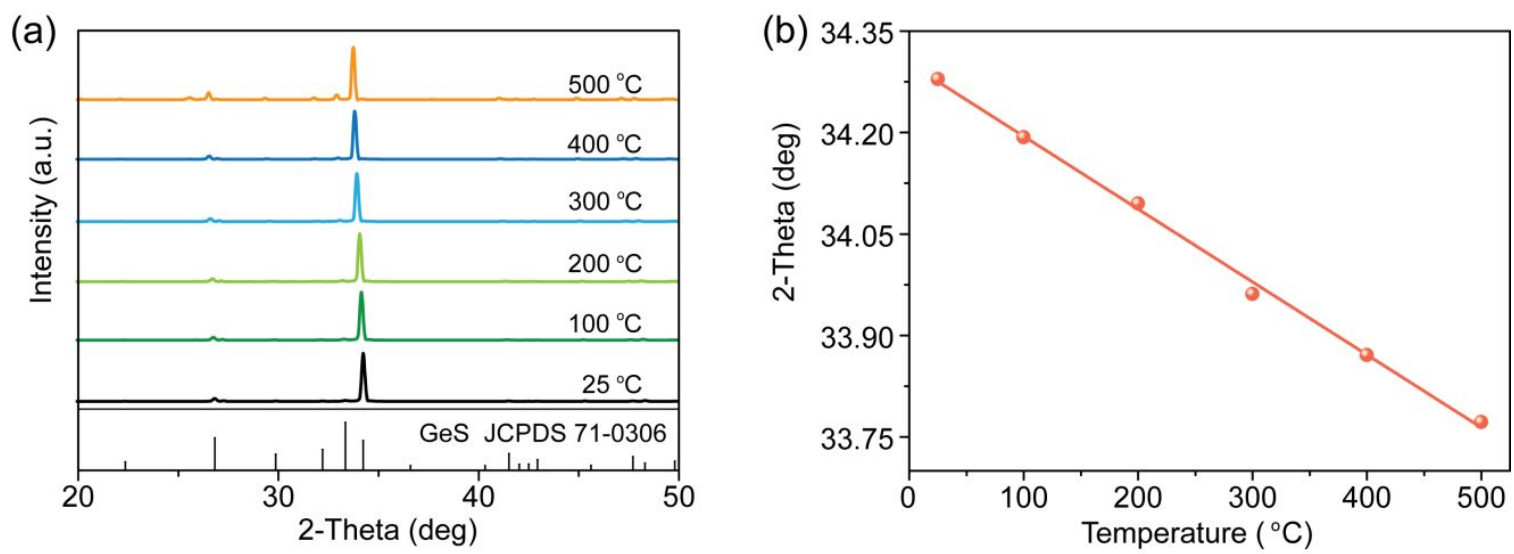

Figure S3. (a) Temperature-dependent XRD patterns of crystalline GeS powder from 25 to $500{ }^{\circ} \mathrm{C}$. (b) Measured (400) peak positions of crystalline GeS powder from from 25 to $500{ }^{\circ} \mathrm{C}$. 


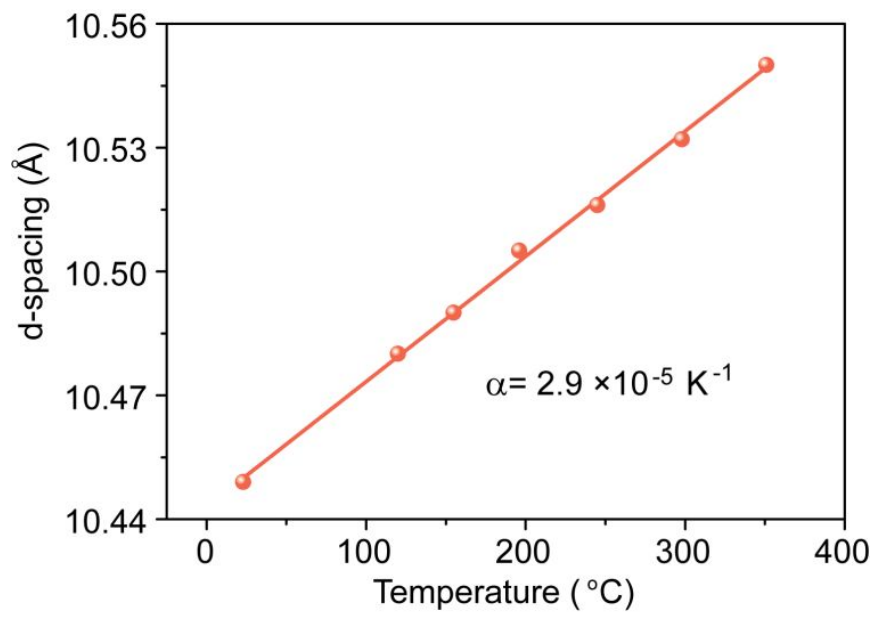

Figure S4. The lattice expansion of GeS as a function of temperature according to a previous report. ${ }^{[7]}$ 


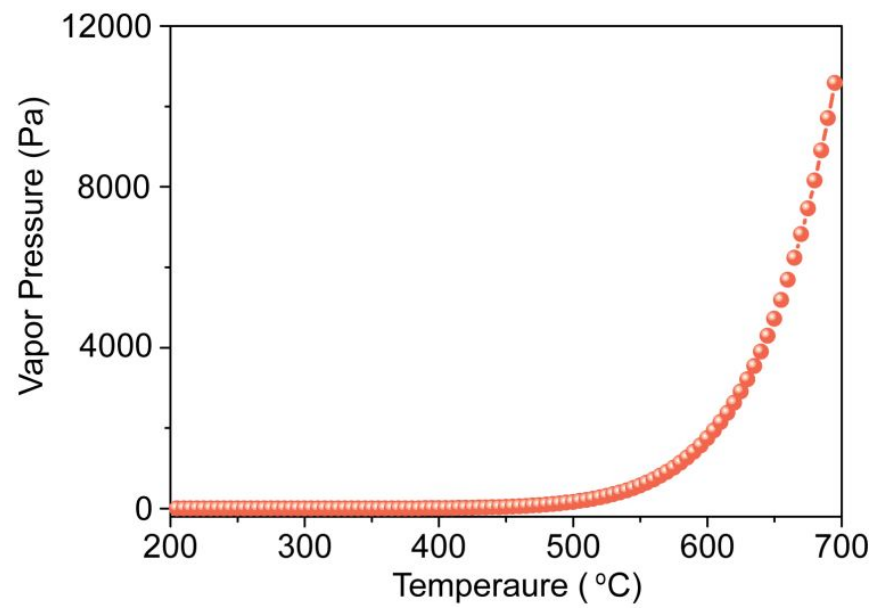

Figure S5. Temperature-dependent vapor pressure of GeS in the temperature range from 200 to $700{ }^{\circ} \mathrm{C}$ according to a previous report. ${ }^{[8]}$ 
(a)

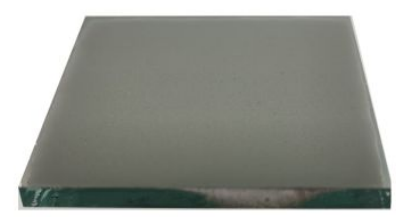

High temperature

(d)

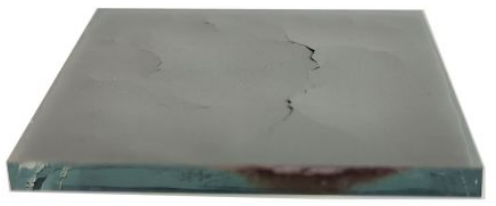

Room temperature (b)

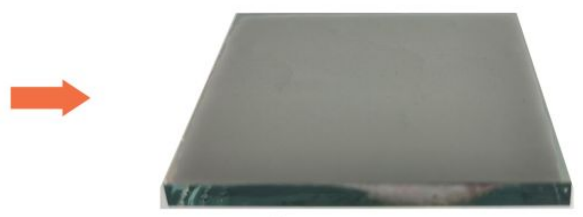

Cooling process

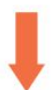

(c)

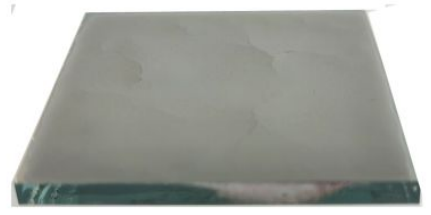

Cooling process

Figure S6. Photographs of GeS film deposited on Mo/glass substrate measured during the cooling process. 


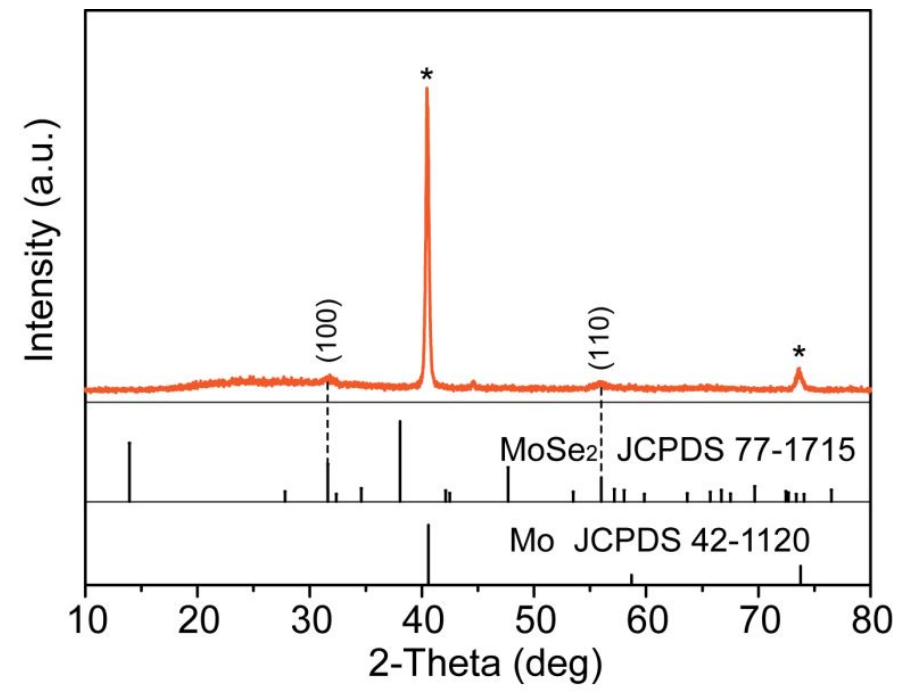

Figure S7. XRD pattern of $\mathrm{MoSe}_{2}$ layer on top of Mo substrate. 


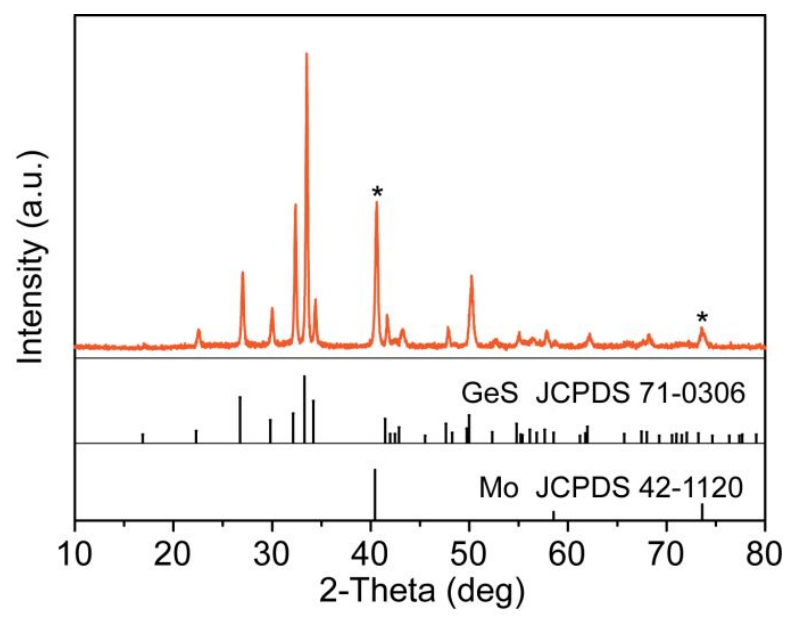

Figure S8. XRD pattern of GeS film deposited on $\mathrm{MoSe}_{2} / \mathrm{Mo}$ substrate. 


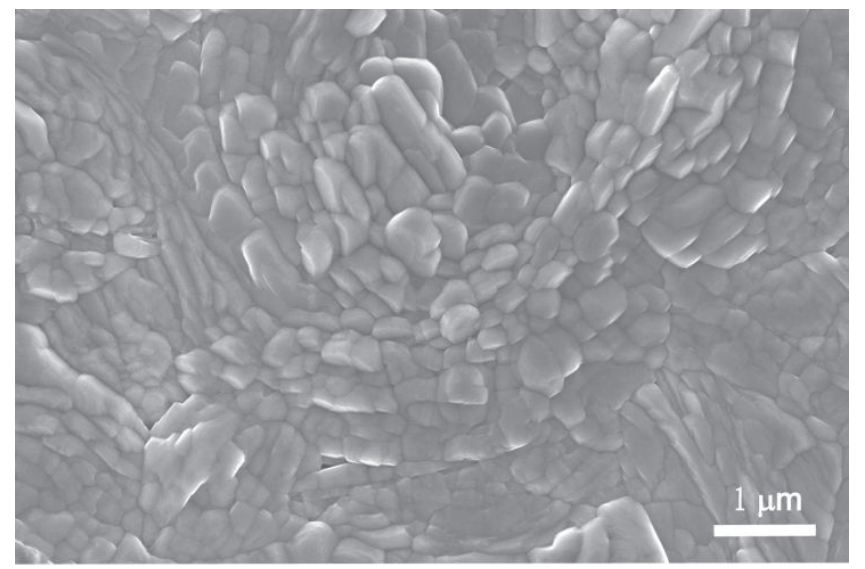

Figure S9. Top-view SEM image of GeS film. 
(a)

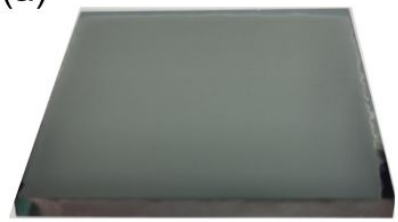

High temperature

(d)

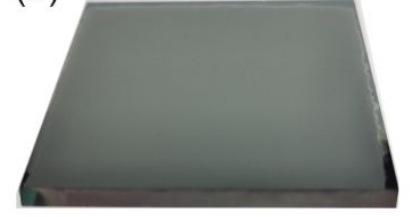

Room temperature (b)

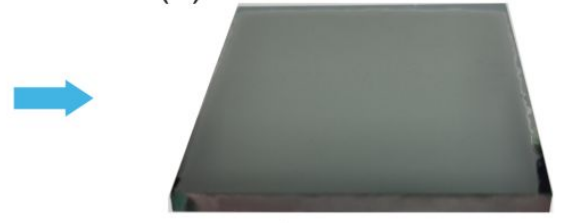

Cooling process

(c)

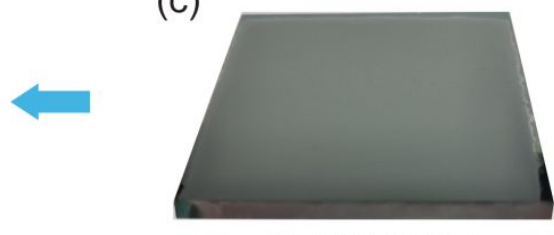

Cooling process

Figure S10. Photographs of GeS film deposited on $\mathrm{MoSe}_{2} / \mathrm{Mo} / \mathrm{glass}$ substrate measured during the cooling process. 


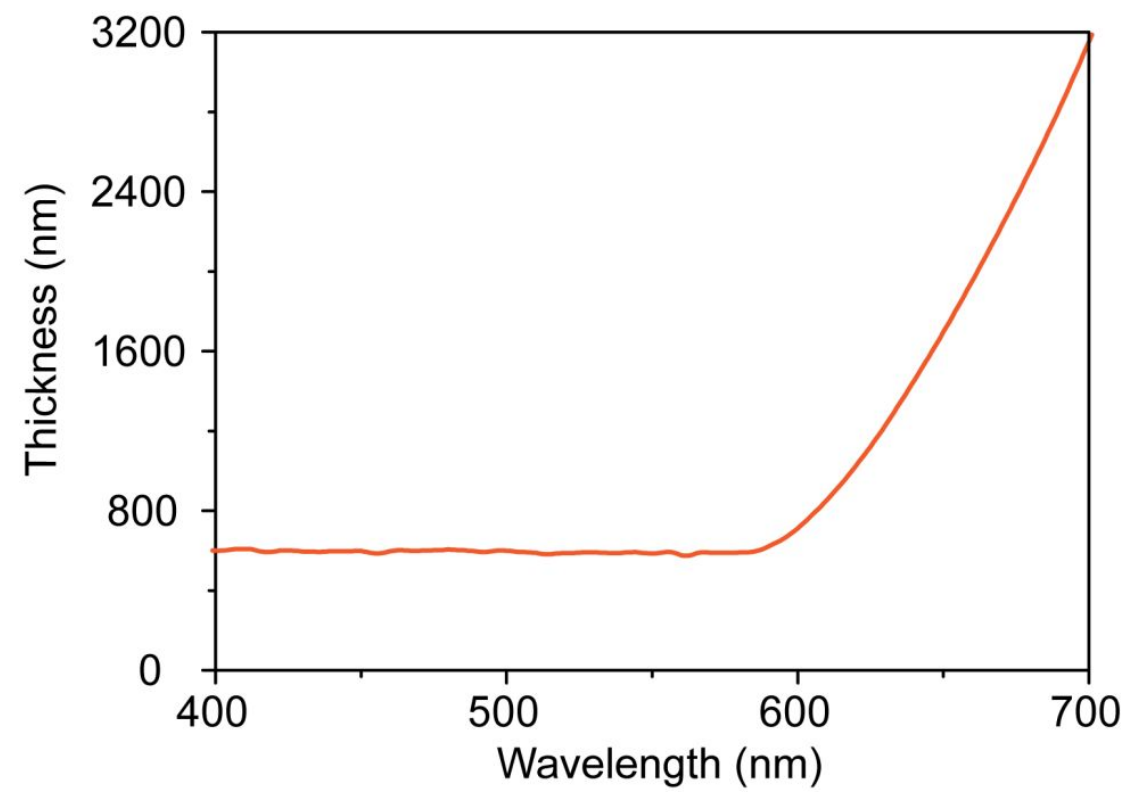

Figure S11. Wavelength-dependent minimum thickness of GeS film for full absorption. 
(a)

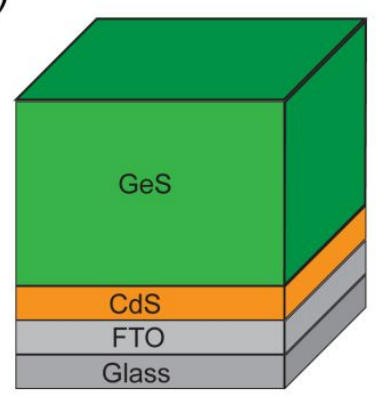

(b)

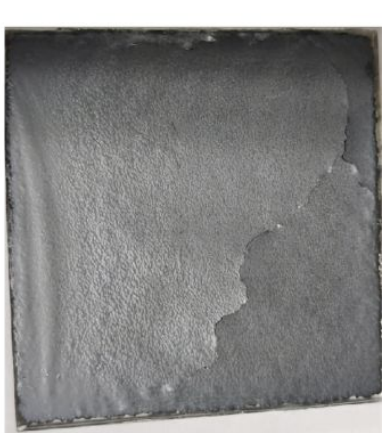

Figure S12. (a) Schematic and (b) photograph of GeS thin film deposited on Glass/FTO/CdS substrate. 


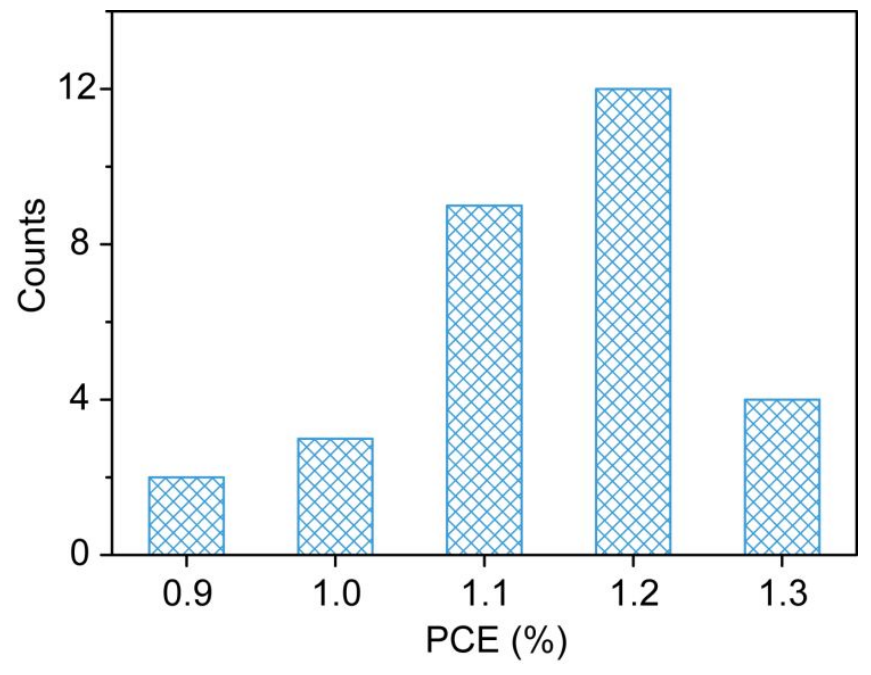

Figure S13. Histogram of device efficiencies obtained from 30 devices. 
Table S1. Orbitally dependent ionic radii as defined by Bloch and Simons. Radii are given in atomic units. ${ }^{[9]}$

\begin{tabular}{lllllll}
\hline Element & $\mathrm{Ge}$ & $\mathrm{Sn}$ & $\mathrm{Pb}$ & $\mathrm{S}$ & $\mathrm{Se}$ & $\mathrm{Te}$ \\
\hline$r_{\mathrm{s}}$ & 0.64 & 0.76 & 0.72 & 0.53 & 0.59 & 0.69 \\
$r_{\mathrm{p}}$ & 0.92 & 1.07 & 1.09 & 0.66 & 0.76 & 0.89 \\
\hline
\end{tabular}

Table S2. A summary of stoichiometries depicted in Figure 1a.

\begin{tabular}{clllllllll}
\hline Material & $\mathrm{GeTe}$ & $\mathrm{GeSe}$ & $\mathrm{GeS}$ & $\mathrm{SnTe}$ & $\mathrm{SnSe}$ & $\mathrm{SnS}$ & $\mathrm{PbTe}$ & $\mathrm{PbSe}$ & $\mathrm{PbS}$ \\
\hline$r_{\pi}^{-1}$ & 2.08 & 2.22 & 2.44 & 1.96 & 2.08 & 2.27 & 1.75 & 1.85 & 2.00 \\
\hline
\end{tabular}




\section{References in Supporting Information}

[1] D.-J. Xue; S.-C. Liu; C.-M. Dai; S. Chen; C. He; L. Zhao; J.-S. Hu; L.-J. Wan. GeSe thin-film solar cells fabricated by self-regulated rapid thermal sublimation. J. Am. Chem. Soc. 2017, 139, 958.

[2] S.-C. Liu; Z. Li; J. Wu; X. Zhang; M. Feng; D.-J. Xue; J.-S. Hu. Boosting the efficiency of GeSe solar cells by low-temperature treatment of p-n junction. Sci. China Mater. 2021, DOI: 10.1007/s40843-020-1617-X.

[3] J.P. Perdew; K. Burke; M. Ernzerhof. Generalized gradient approximation made simple. Phys. Rev. Lett. 1996, 77, 3865.

[4] G. Kresse; J. Hafner. Ab initio molecular-dynamics simulation of the liquid-metal amorphous-semiconductor transition in germanium. Phys, Rev. B. 1994, 49, 14251.

[5] G. Kresse; J. Furthermuller. Effect of Er doping on the electronic structure of optical properties of ZnO. Phys. Rev. B 1996, 54, 11169.

[6] H.J. Monkhorst; J.D. Pack. Special points for Brillouin-zone integrations. Phys. Rev. B 1976, 13,5188 .

[7] H. Wiedemeier; P.A. Siemers. The thermal expansion of GeS and GeTe. Z. anorg. allg. Chem. 1977, 431, 299.

[8] C. Hirayama. Thermodynamic properties of solid monoxides, monosulfides, monoselenides, and monotellurides of Ge, Sn, and Pb. J. Chem. Eng. Data 1964, 9, 65.

[9] J.R. Chelikowsky; J.C. Philips. G. K. Quantum-defect theory of heats of formation and structural transition energies of liquid and solid simple metal alloys and solid simple metal alloys and compounds. Phys. Rev. B. 1978, 17, 2453. 